\title{
A Chemistry Scale-up Exercise
}

\author{
Summary \\ Second year students take a bench scale synthesis and work out how to scale it up. \\ They simulate the process with salt and water to determine practical handling \\ problems and thereby develop a set of instructions for process operators.
}

Subject area: Chemistry

\section{Description}

There is considerable activity in the chemical industry in making relatively small amounts of chemical products for special purposes, say from a kilogram to a few tonnes. Many companies synthesise materials in single batches or short campaigns as required. To scale up a synthesis from gram (or less) to kilogram or more brings practical problems of material handling, safety and general organization which are not addressed by standard laboratory classes in chemistry or chemical engineering. Nevertheless, it is a task the graduate might well face in industry.

For the past five years, undergraduates in Chemical Engineering, and the Joint Honours degree Chemical Engineering and Chemistry, at the University of Sheffield have carried out practical exercises based on this problem. The long version actually involves synthesis of a kilogram of product, using similar quantities of toxic, corrosive, flammable chemicals. This requires suitable facilities and has some cost. However the short version uses nothing other than salt and water, and thus could be carried out with minimal cost and safety concerns, while still addressing much of the educational value.

\section{Activity}

Short Laboratory ( 3 to 5 hours)

Groups of 3 students are provided with a bench-scale synthesis from a textbook and directed to scale it up. We have a 50-litre flask and mantle, with stirrer, feed funnel, cooling coils and condenser. This could be adapted to distillation, or material could be transferred to an adjacent distillation apparatus. There is also a filter press. However, much of the educational aims could be achieved with 2-litre or 5-litre vessels and a large Buchner filter, and this report is made for departments who wish to consider the more modest scale.

The first task is to determine the minimum and maximum working volume of the main vessel, and compare this with the amount which occurs in the bench scale. A large spherical vessel needs a certain minimum volume for the stirrer to be effective, but should not be filled to more than two-thirds full, particularly if is to be refluxed. A good scale factor will give convenient quantities for measuring out on the large scale. Students should understand what each component is for, and the chemistry involved: it is not enough to blindly multiply. Reagents may need to be present in a ratio determined by stoichiometry - others such as a solvent may be less critical. 
The students then make up a stepwise set of instructions. There should only be one action per step and it should be specific. E.g. "Switch on the agitator." "Slowly add 2 litres of $35 \%$ aqueous $\mathrm{HCl}$." "Switch on the heating coils." "Heat up to $60{ }^{\circ} \mathrm{C}$." "Switch off the heating coils."

The next stage is to mimic the process, but using sodium chloride in place of any solid and water in place of any liquid. The aim is to determine how long it will take to carry out each step and the practical implications. This may require some subsidiary tests. If the original instructions say "reflux for 3 hours" there is no need to actually do this: just write down this step and go on to the next one. However, how long does it take to get to boiling point? This can be estimated by extrapolating from a heating curve (taking into account the boiling point of the solvent used). Ideally, students should correct for the different specific heat of the solvent compared with water.

However, it must be stressed that a key feature is practical handling. Students are often surprised that sodium chloride does not instantly dissolve, or that wet solid is sticky. Successful scale-up may actually require things to be done in a different order. Typically, to dissolve solid requires there to be sufficient liquid for the stirrer to be effective and then to add the solid slowly so that it is dispersed.

If salt and water cause difficulty, what about corrosive chemicals such as phosphorus pentoxide or benzyl chloride? How could the handling be modified for safety? In a 5-hour lab the students may actually act out these procedures; in a 3-hour lab it is a matter for discussion.

The student's target achievement is a revised set of instructions which include safety requirements and a reasonable estimate of the time for each step, including measuring out of chemicals. The laboratory report includes this, plus the work and thought they put in to get it. As an option, they may be required to carry out a COSHH assessment.

Note that with some syntheses it may not be possible to simulate all of it because of lack of equipment or time. The demonstrator should suggest a suitable starting and/or stopping point in the synthesis. The other steps should be estimated.

Long Laboratory ( 3 sessions of 3 to 8 hours)

In this the students actually carry out the synthesis as follows. In the first session, they carry out the bench scale following the laboratory recipe, but observe it carefully and make notes. This is where it is helpful to have three people. They may also carry out subsidiary tests, noting matters such as temperature rise, emission of fumes, difficulty in dissolving.

They prepare a stepwise protocol and go through it in the second session, using sodium chloride and water. This enables them to sort out problems of organization and timing. (Or indeed, miscalculation of volume!) They prepare a revised plan and a COSHH assessment, which has to be agreed by a member of staff.

In the third session, the students actually carry out the synthesis. This can provide a great sense of achievement or possibly a harsh lesson! Naturally they have to be well 
New Directions in the Teaching of Physical Sciences

supervised and fulfil all necessary safety requirements. It may be appropriate for a member of technical staff to carry out certain operations.

\section{Further comments}

Clearly, few departments will have the resources to carry out pilot scale chemistry.

We only provide the long lab experience to 12 students a year. However, scale-up is a useful art to appreciate and can be readily simulated with low cost and low risk using salt and water. Current chemical laboratory practice commonly involves semi-micro or less, so even dissolving up tens of grams of salt in a litre of water may be worthwhile. Certainly, thinking about what is happening both chemically and physically would be helpful for students intending to work in the manufacturing part of industry.

\section{Author(s)}

Martin J. Pitt, University of Sheffield

\section{Contact details}

Martin J. Pitt

Chemical and Process Engineering

University of Sheffield

m.j.pitt@sheffield.ac.uk 